\title{
Interpretation of x-ray-absorption dichroism experiments
}

\author{
P. Strange \\ Department of Physics, Keele University, Keele, Staffordshire ST5 5BG, United Kingdom \\ B. L. Gyorffy \\ Department of Physics, Bristol University, Tyndall Avenue, Bristol BS8 1TL, United Kingdom
}

(Received 15 June 1995)

\begin{abstract}
A rule is derived to use $\mathrm{x}$-ray magnetic circular dichroism spectra to extract the magnetic moment of the conduction-band states with $j=l-\frac{1}{2}$ separately from those with $j=l+\frac{1}{2}$ as a function of energy. This quantity is straightforward to determine from the electronic band structure. The rule is illustrated with an application to pure iron and to the random substitutional alloy $\mathrm{Fe}_{80} \mathrm{Co}_{20}$.
\end{abstract}

The observation of magnetic circular dichroism in $x$-ray absorption (MXCD) by Schütz et al. ${ }^{1}$ and subsequent experimental work ${ }^{2}$ has led to MXCD becoming a very popular and unique probe of magnetism in transition and rare-earth metallic systems. Theoretical understanding of MXCD has also made significant progress in recent years starting from both a localized $^{3}$ and itinerant ${ }^{4,5}$ view of magnetism. This has culminated in the recent exceedingly powerful, yet simple, MXCD sum rules ${ }^{6-8}$ which have shown that MXCD can be used to measure the spin and orbital contributions to the magnetic moment separately. The sum rules are derived from a single ion model and it has been shown that, although approximate, they are often accurate to better than $5 \% .^{9,10}$ More recently, Ankudinov and $\mathrm{Rehr}^{11}$ have rederived the sum rules using Fermi's golden rule within the independent electron approximation. There is some uncertainty in such a calculation as the sum rule requires an energy integration which is cut off at some judiciously chosen, but ultimately arbitrary value. Recently it has been shown that there are some circumstances where the sum rules are of limited value. A particular example is on some transition metal surfaces where errors of up to $50 \%$ occur. ${ }^{12}$ However, even in such cases the ratio of the spin to orbital moments can be determined to an accuracy of about $10 \%$. One limitation of the sum rules derived so far is that they are based on nonrelativistic quantum theory with relativistic corrections to order $1 / c^{2}$, not on Dirac's relativistic quantum theory. In this paper we derive an alternative rule for interpreting MXCD experiments based on an itinerant, rather than localized approach within a fully relativistic quantum mechanical framework. The rule enables us to determine directly the total magnetic moment of the conduction band $j=l-\frac{1}{2}$ states separately from the magnetic moment of the $j=l+\frac{1}{2}$ states where $l$ is defined by dipolar transitions. Furthermore this can be determined at every energy, so in principle no energy integration is required. In principle this is a significant advance because dichroism curves often contain considerable structure as a function of energy, which is not described by the sum rules, however, broadening effects may well lead to the rule being more applicable in integral form where the total magnetic moment of the occupied $j=l-\frac{1}{2}$ states can be determined.
Our starting point for this calculation is the golden rule for the transition probability per unit time:

$$
W_{c}^{\lambda}=\frac{2 \pi}{\hbar} \sum_{f}\left|\left\langle\psi_{f}\left|X_{q}^{\lambda}\right| \psi_{i}\right\rangle\right|^{2} \Theta\left(E_{F}-E_{c}-\hbar \omega\right),
$$

where the summation is over all possible final states, and $\Theta(E)$ is zero for $E<0$ preventing the filling of states below the Fermi energy $E_{F} . E_{c}$ is the energy of the core electron being excited and $\omega$ is the frequency of the incident photon. $\psi_{i}$ and $\psi_{f}$ are the wave function describing the system in its initial and final states, respectively. In a density functional approach these are commonly replaced by single particlelike wave functions. ${ }^{13}$ The photon-electron interaction operator $X_{q \lambda}$ is defined as

$$
X_{q \lambda}(\mathbf{r}) \equiv-e \underline{\boldsymbol{\alpha}} \cdot \mathbf{A}(\mathbf{r})=-e \underline{\boldsymbol{\alpha}} \cdot \hat{\boldsymbol{\epsilon}}^{(\lambda)} e^{i \mathbf{q} \cdot \mathbf{r}},
$$

where $\mathbf{A}(\mathbf{r})$ is the vector potential associated with the incident radiation and $\underline{\boldsymbol{\alpha}}$ are Dirac matrices in the standard representation. It is in the $\hat{\boldsymbol{\epsilon}}^{(\lambda)}$ that we distinguish between different polarizations of the incident radiation. The core wave function can be separated into radial and angular parts: ${ }^{14}$

$$
\psi_{\kappa_{c}}^{m_{j}}(\mathbf{r})=\sum_{\kappa_{c}^{\prime}=\kappa_{c},-\kappa_{c}-1}\left(\begin{array}{cc}
g_{\kappa_{c}, \kappa_{c}^{\prime}}^{m_{j}}(r) & \chi_{\kappa_{c}^{\prime}}^{m_{j}(\hat{\mathbf{r}})} \\
i f_{\kappa_{c}, \kappa_{c}^{\prime}}^{m_{j}}(r) & \chi_{-\kappa_{c}^{\prime}}^{m_{j}}(\hat{\mathbf{r}})
\end{array}\right)
$$

and the final state wave function can be written as a linear combination of atomic orbitals

$$
\psi_{f}(\mathbf{r})=\sum_{\kappa, m_{j}} c_{\kappa, m_{j}} \psi_{\kappa}^{m_{j}}(\mathbf{r})
$$

where

$$
\psi_{\kappa}^{m_{j}}(\mathbf{r})=\sum_{\kappa^{\prime}=\kappa,-\kappa-1}\left(\begin{array}{ll}
g_{\kappa, \kappa^{\prime}}^{m_{j}}(r) & \chi_{\kappa^{\prime}}^{m_{j}(\hat{\mathbf{r}})} \\
i f_{\kappa, \kappa^{\prime}}^{m_{j}}(r) & \chi_{-\kappa^{\prime}}^{m_{j}}(\hat{\mathbf{r}})
\end{array}\right) .
$$

Here the $\chi_{\kappa}^{m_{j}}(\hat{\mathbf{r}})$ are the usual spin angular functions, and we work in the $\left(\kappa, m_{j}\right)$ representation throughout. ${ }^{15}$ The $\underline{\alpha}$ are odd matrices and so couple the large and small parts of the 

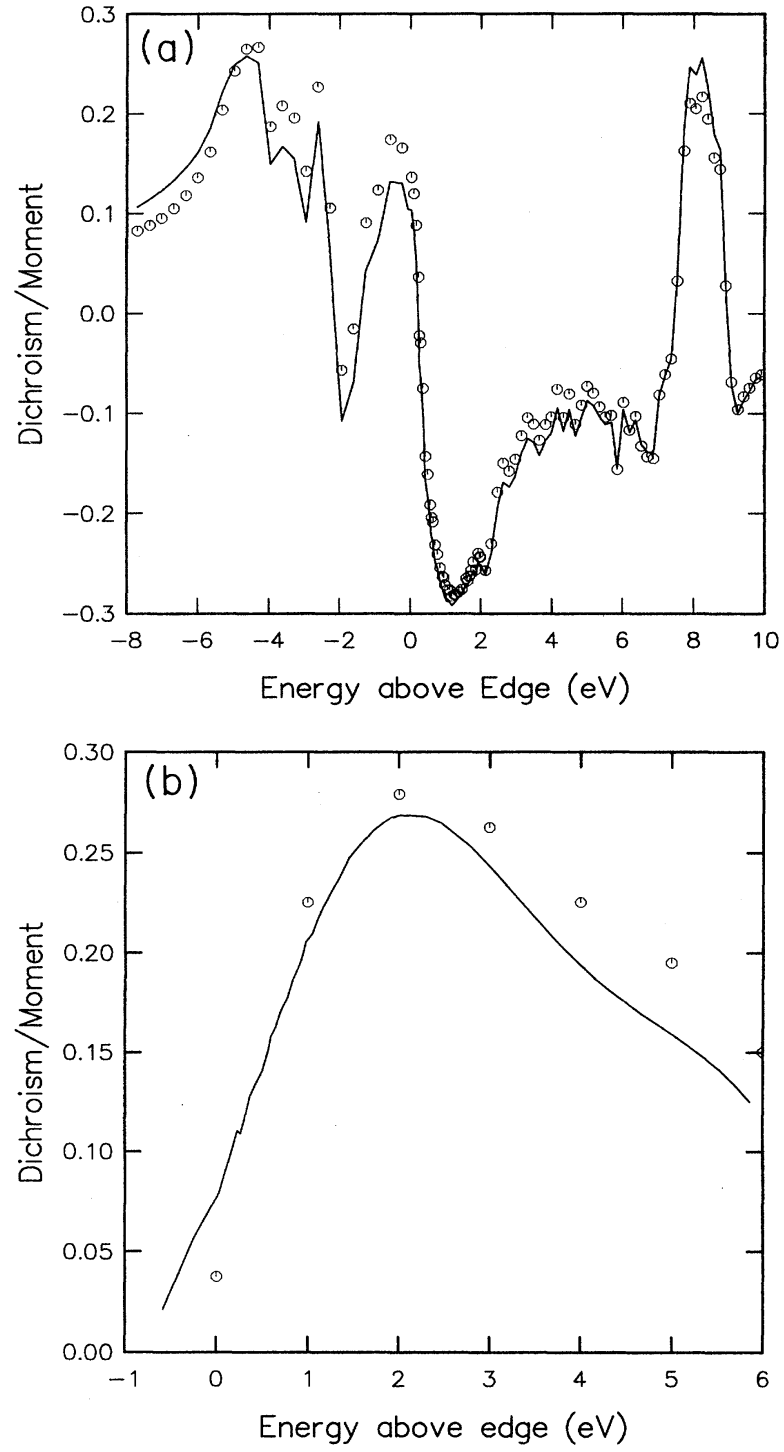

FIG. 1. (a) Illustration of Eq. (5) for pure iron. The full line is the right-hand side of Eq. (5) calculated for the $j=\frac{3}{2} d$-electrons. The points are the left-hand side of Eq. (5) calculated at the $L_{2}$ edge. (b) The full line here is the right-hand side of Eq. (5) calculated for the $j=\frac{3}{2} d$-electronic states above $E_{F}$. This curve has been convoluted with a Gaussian of width $1.2 \mathrm{eV}$ to simulate all broadening mechanisms and has been inverted to make direct contact with experiment. The points are evaluated directly from the experimental results on the $L_{2}$ edge of iron in Ref. 2 .

wave functions only. Substituting Eqs. (2) and (3) into (1) and performing the angular integrals leads to the usual absorption selection rules which depend upon the polarization of the incident photon. ${ }^{4}$ The radial matrix elements also have to be evaluated. In common with Refs. 6-8 we find that the radial matrix elements are virtually independent of $m_{j}$. Also the $\kappa_{c}^{\prime}=-\kappa_{c}-1$ and $\kappa^{\prime}=-\kappa-1$ terms in the summations in (3a) and (3c) lead to radial matrix elements which are much smaller than the terms with $\kappa_{c}^{\prime}=\kappa_{c}$ and $\kappa^{\prime}=\kappa$ so they can be neglected for our purposes. Hence the radial parts of the wave function can be labeled with just a single $\kappa$ subscript. If we use the notation:

$$
\begin{aligned}
& R 1_{\kappa_{c}, \kappa}=\int r^{2} g_{\kappa_{c}}(r) f_{\kappa}(r) d r, \\
& R 2_{\kappa_{c}, \kappa}=\int r^{2} f_{\kappa_{c}}(r) g_{\kappa}(r) d r,
\end{aligned}
$$

we find that

$$
R 2_{\kappa_{c},-\kappa-1} \approx R 2_{\kappa_{c}, \kappa} \approx-R 1_{\kappa_{c}, \kappa} \approx-R 1_{\kappa_{c},-\kappa-1},
$$

and in the cases we have examined these are at least an order of magnitude larger for the dipolar matrix elements than for any other terms. Equation (4c) is an approximation of the same order as a nonrelativistic approximation. Making these approximations in our evaluation of Eq. (1) facilitates the mathematics considerably and we can calculate the dipole transition probability from any absorption edge to any allowed state. If we restrict ourselves to transitions of the type $l_{c} \rightarrow l_{c}+1$ (this is not a great restriction in practice as the large majority of experiments work on $p \rightarrow d$ or $d \rightarrow f$ transitions), we find the following:

$$
\begin{aligned}
\frac{W_{j_{c}-}^{+1}-W_{j_{c}-}^{-1}}{W_{j_{c}-}^{+1}+W_{j_{c}-}^{-1}+W_{j_{c}-}^{0}} & \approx-\frac{1}{j_{-}} \frac{\sum_{m_{j}}\left|c_{j_{-}, m_{j}}\right|^{2} m_{j}}{\sum_{m_{j}}\left|c_{j_{-}, m_{j}}\right|^{2}} \\
& =-\frac{1}{j_{-}} \frac{\left\langle j_{z_{-}-}\right\rangle}{\left\langle N_{j-}\right\rangle} .
\end{aligned}
$$

Equation (5) is the central result of this paper. It allows us an alternative interpretation of dichroism experiments on the $j_{c-}=l_{c}-\frac{1}{2}$ edge in transition and actinide metal systems. From an experiment at the $j_{c}$ - edge only, the total magnetic

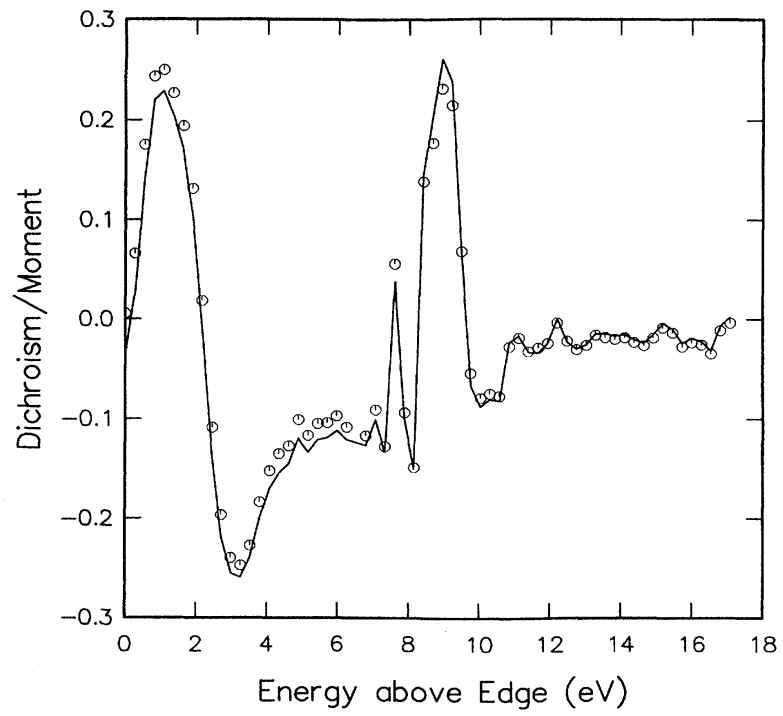

FIG. 2. Illustration of Eq. (5) for the random substitutional alloy $\mathrm{Fe}_{80} \mathrm{Co}_{20}$. The full line is the right-hand side of Eq. (5) calculated for the $j=\frac{3}{2} d$-electrons on the iron site. The points are the left-hand side of Eq. (5) calculated at the iron $L_{2}$ edge. 


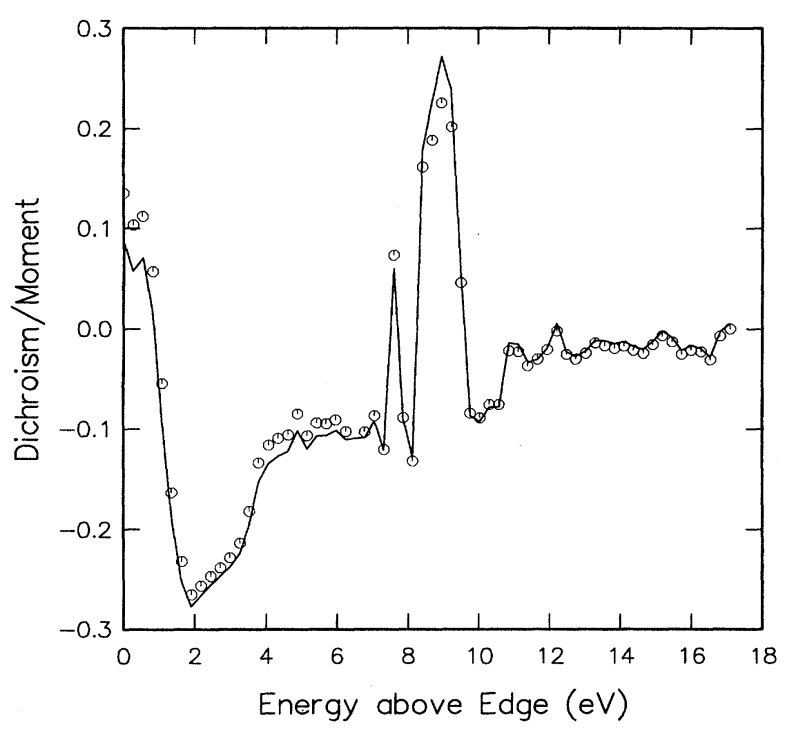

FIG. 3. Illustration of Eq. (5) for the random substitutional alloy $\mathrm{Fe}_{80} \mathrm{Co}_{20}$. The full line is the right-hand side of Eq. (5) calculated for the $j=\frac{3}{2} d$ electrons on the cobalt site. The points are the lefthand side of Eq. (5) calculated at the cobalt $L_{2}$ edge.

moment per electron of the $j=l-\frac{1}{2}$ states in the conduction band can be found energy by energy. Furthermore, in analogy with the already known sum rules ${ }^{6-8}$ we can integrate Eq. (5) from the Fermi energy up to the top of the band to find the total magnetic moment of the $j=l-\frac{1}{2}$ electronic states in that region. As a completely full band can have no net magnetic moment the result of the integration is just the negative of the total magnetic moment per electron of the filled part of the band. Equation (5) is dimensionless and so the absorption rate can be determined in arbitrary units. For $\left\langle N_{j-}\right\rangle$ we can simply use the density of states and for $\left|c_{j-, m_{j}}\right|^{2} m_{j}$ we use the $m_{j}$ quantum number multiplied by the $m_{j}$ decomposed contribution to the $j=l-\frac{1}{2}$ part of the density of states. Both these quantities are easily obtainable from a standard band theory calculation. However, in the past there has been no way of determining them independently of the $j=l+\frac{1}{2}$ contributions experimentally. Equation (5) has the minor advantage over the earlier sum rules ${ }^{6,7}$ that it only requires the approximation (4c) over one edge, not over both the $j_{c}=l_{c} \pm \frac{1}{2}$ edges. Finally, it does not seem to be so straightforward to find a similar rule using excitations from the $j=l_{c}+\frac{1}{2}$ edge. A consequence of this is that we are restricted to materials where the width of the empty part of the conduction band is smaller than the spin-orbit splitting of the core level. In practice this is not a severe restriction. Even in pure iron this is easily satisfied.

To illustrate the use of Eq. (5) we have applied it to the $L_{2}$ edge in pure iron using the theories of Strange and Ebert. $^{16,4}$ Figure 1(a) shows the result. The full line is the right-hand side of Eq. (5) and the points are the left-hand side. Here, for illustrative purposes we have applied the rule to the whole band structure, although, obviously, an MXCD experiment could only probe the region above zero on this figure. Clearly there is excellent quantitative agreement between both sides of Eq. (5), even in the low-energy region where the $d$-electron contribution to the density of states is small. In Fig. 1(b) we show the right-hand side of Eq. (5) compared directly with experiment ${ }^{2}$ for the $L_{2}$ edge in Fe. To make this comparison we fitted energy scale such that the edges in both theory and experiment coincided and performed a Gaussian broadening of width $1.2 \mathrm{eV}$ on the calculated values to take account of the various broadening mechanisms present in the experiment. This shows clearly the utility of the method either for predicting dichroism spectra from theory, or for using the experimental results to evaluate features of the band structure accurately.

We have made two further applications of the rule, to the $L_{2}$ edge of both $\mathrm{Fe}$ and $\mathrm{Co}$ in the random substitutional alloy $\mathrm{Fe}_{80} \mathrm{Co}_{20}$. The results are shown in Figs. 2 and 3 respectively. Clearly the agreement here is at least as good as in the pure iron case, and many details are well reproduced by the rule. For example, the peak in the dichroism at about $8 \mathrm{eV}$ above the Fermi energy in pure iron has split into two when alloyed with $20 \%$ Co. The magnetic moment per $j=l-\frac{1}{2}$ electron clearly reproduces this.

The power and utility of Eq. (5) can only be proven by comparison between experiment and the theory, and as with all such rules is subject to the limitations of the experiment. In any real experiment much of the detail on figures such as those shown is washed out by the various mechanisms such as core-hole relaxation and instrumental broadening. Therefore only some broadened form of the right-hand side of Eq. (5) can be obtained directly from experiment. Nonetheless if we integrate both sides of (5), the total moment per $j=l$ $\frac{1}{2}$ electron can be obtained, and we believe this should be obtainable from experiment. On the other hand, as we have shown, the right-hand side of Eq. (5) can be obtained rather easily from band theory. Broadening this quantity by convoluting it with a suitable function should reproduce the energy dependence of the dichroism curve.

Some approximations have gone into our deviation. In particular we have ignored dipole transitions from $l \rightarrow l-1$ and we have approximated the initial core state as a pure eigenstate defined by quantum numbers $\left(\kappa, m_{j}\right)$, which is equivalent to ignoring the polarization of the core hole. Furthermore we have not gone beyond the dipole approximation in evaluating our matrix elements. The extent to which the experimental spectra do not agree with Eq. (5) may be used to investigate their validity of these assumptions.

In summary then, we have derived a rule for the interpretation of MXCD experiments which relates the dichroic signal to the magnetic moment of the $j=l-\frac{1}{2}$ electrons in the solid, separately from the magnetic moment of the $j=l+\frac{1}{2}$ electrons at every energy. The rule means that it is possible to separate these two quantities experimentally. Hence this rule offers a deeper comparison between experiment and theory than has been possible previously. This rule is applicable where an itinerant view of the magnetism is appropriate. It is complementary to the previously derived sum rules, ${ }^{6,7}$ and essentially offers an alternative representation of the ground state magnetic properties.

Now that we have derived the relation (5) the next step is to test its range of validity by applying it to a wide range of materials and in more general circumstances, such as at magnetic surfaces. One further possibility is that this rule will also be applicable to materials which exhibit dichroism, because the symmetry is broken not by magnetism, but by a crystal structure without a center of inversion. We hope that this paper will stimulate such investigations. 
${ }^{1}$ G. Schütz, W. Wagner, W. Wilhelm, P. Kienle, R. Zeller, R. Frahm, and C. Materlik, Phys. Rev. Lett. 58, 737 (1987).

${ }^{2}$ N. V. Smith, C. T. Chen, F. Sette, and L. F. Mattheiss, Phys. Rev. B 46, 1023 (1992).

${ }^{3}$ B. T. Thole, G. van der Laan, and G. A. Sawatzky, Phys. Rev. Lett. 55, 2086 (1985).

${ }^{4}$ H. Ebert, P. Strange, and B. L. Gyorffy, Z. Phys. B Condens. Matter 73, 77 (1988).

${ }^{5}$ See, for example, H. Ebert, B. Drittler, P. Strange, R. Zeller, and B. L. Gyorffy, in The Effects of Relativity in Atoms, Molecules and the Solid State, edited by S. Wilson, I. P. Grant, and B. L. Gyorffy (Plenum, New York, 1991).

${ }^{6}$ B. T. Thole, P. Carra, F. Sette, and G. van der Laan, Phys. Rev. Lett. 68, 1943 (1992).

${ }^{7}$ P. Carra, B. T. Thole, M. Altarelli, and X.-D. Wang, Phys. Rev. Lett. 70, 694 (1993).
${ }^{8}$ M. Altarelli, Phys. Rev. B 47, 597 (1993).

${ }^{9}$ R. Wu, D. Wang, and A. J. Freeman, Phys. Rev. Lett. 71, 3581 (1993).

${ }^{10}$ P. Strange, J. Phys. C 6, L491 (1994).

${ }^{11}$ A. Ankudinov and J. J. Rehr, Phys. Rev. B 51, 1282 (1995).

${ }^{12}$ R. Wu and A. J. Freeman, Phys. Rev. Lett. 73, 1994 (1994).

${ }^{13}$ P. J. Durham, in The Electronic Structure of Complex Systems, edited by P. Phariseau and W. M. Temmerman (Plenum, New York, 1984).

${ }^{14}$ P. Strange, J. B. Staunton, and B. L. Gyorffy, J. Phys. C 17, 3355 (1984).

${ }^{15}$ M. E. Rose, Relativistic Electron Theory (Wiley, New York, 1961).

${ }^{16}$ P. Strange, H. Ebert, J. B. Staunton, and B. L. Gyorffy, J. Phys. Condens. Matter 1, 2959 (1989). 